\title{
Adaptive Sliding Mode Control Design of a SCARA Robot Manipulator System Under Parametric Variations
}

\author{
F. Adelhedi*, A. Jribi, Y. Bouteraa and N. Derbel \\ University of Sfax, Sfax Engineering School, Control and Energy Management Laboratory (CEM-Lab), Tunisia
}

Received 14 August 2015; Accepted 15 December 2015

\begin{abstract}
- The sliding mode control (SMC) has yet proven its efficiency through several theoretical researches. Indeed, the robotic field is recognized as one of the main SMC portals on practical implementations. The interest of this work consists in testing the SMC robustness and its reliability versus the parameters variation and model uncertainties.

In this paper, an algorithm for trajectory tracking task of robot manipulators based on a SMC has been proposed. Then, aiming to deal with the presence of disturbances and parametric modeling uncertainties, the adopted control law has been extended to an adaptive SMC version based integral sliding surface, where the selection of the parameters adaptation law has been detailed. It has been proven that the adaptive control design can stabilize both position and velocity of the system, where the explicit use of the system dynamic model becomes no longer required. Simulation results performed on a SCARA robot manipulator reveal improving control acting clearly denoted by the introduction of the adaptive control design.
\end{abstract}

Keywords: Sliding mode control, nonlinear systems, adaptive control, parametric variations, robot manipulators.

\section{Introduction}

Robotic systems frequently present intense non linearities, and are always subject to model parameters uncertainties and disturbances. Such factors make difficulties in system measurements and transform their control to a hard task. For this reason, and in order to ensure the certain convergence of the system to the requested state condition, a high level control is often required. Furthermore, structuring Lyapunov functions has been ever considered a fundamental matter in stabilizing system control.

In this context, sliding mode control that is known as a variable structure Control (VSC), is highly advisable due to its easy implementation and its robustness via uncertainties [8], [7]. In fact, SMC operates in such away that system states are forced to evolve in accordance to a chosen 'sliding manifold' in the state space. Once the targeted sliding surface has been affected, the controlled system turns to successfully reduce and even eliminate parametric uncertainties and external disturbances. In fact, it has potential advantages that can be summarized in two major points. Firstly, the accurate compensation of uncertainties under matching conditions for nonlinear systems is an obvious characteristic leading to external disturbances tackle. The second point concerns the finite-time convergence to the sliding surface [12]. However, and in spite of the advantages previously cited, the discontinuous nature of the sliding mode control induces rise to high

* E-mail address: fatma.abdelhedi@live.com ISSN: 1791-2377 (C) 2015 Kavala Institute of Technology. All rights reserved. frequency oscillations that generate the dangerous chattering phenomenon $[5,6]$.

Aiming to address such impediment in the sliding mode controller, several methods have been suggested [4]. Most of them consist on a continuous approximation of the discontinuous feedback law. A widely known alternative is the use of approximations to the sign function [2, 9]. Saturation, sigmoïdes and hysteresis functions are often used, offering a continuous or smooth control signal. In this case, there will be a trade-off between the smoothness of response and the robustness of closed loop system.

Besides, another difficulty faced by most of the sliding mode controllers is that the upper bound of the system uncertainties must be a priori known which can be hardly specified in several practical implementations. The proposed solution consists in realizing an adaptive SMC design, whose goal is to resolve the unpredictability and the complexity of the process uncertainties, and to overcome therefore such restrictions by the use of estimating adaptation schemes. However, very few results have proposed adaptive approach incorporated into the sliding mode control $[1,11,13]$. By a similar way, this controller combination do not require full information on the bounds of uncertainties and perturbations. The interest is the adaptation of system parameters and the rapid attainment of the right parameters values, with respect to uncertainty effects.

In this paper, a proposed control approach to high frequency oscillations avoidance is developed, by incorporating adaptive control architecture into the SMC architecture. Indeed, adaptive control presents an effective strategy used to address the parameter uncertainty problem.

Besides, the incorporated adaptation law mixed to the Lyapunov based proof investigates the stability of the whole system, with adaptation of system parameters yielding a 
more efficient way to face external perturbations and uncertainties.

This paper is organized as follows. Section 2 presents the modeling of a manipulator robot. In section 3, an algorithm for trajectory tracking based on a SMC is presented. Then, aiming to deal with the presence of disturbances and parametric modeling uncertainties, the adopted control law has been extended to an adaptive SMC design. Finally, in order to evaluate the efficiency of the proposed controllers, section 4 presents simulation results obtained by a SCARA trajectory tracking, of both simple and the adaptive SMC versions. The stability analysis based on Lyapunov approach has been proved.

\section{Modeling}

Let's consider the dynamic model of a SCARA robot system with three degrees of freedom, rigid links, and without joints friction. One of its general representations shows an assembly arm represented subsequently, where the two first are revolute joints, and the third one is a prismatic articulation. The corresponding equation can be expressed as:

$$
M(q) \ddot{q}+C(q, \dot{q}) \dot{q}+G(q)=t
$$

where, for the considered SCARA robot $n=3$ :

- $q \in \mathbb{R}^{n}$ denotes the measured displacements vector,

- $\dot{q} \in \mathbb{R}^{n}$ is the measured velocity vector,

- $\dot{q} \in \mathbb{R}^{n}$ is the articulatory acceleration vector,

- $M(q) \in \mathbb{R}^{n \times n}$ is the inertia matrix, which is symmetric uniformly bounded and positive definite,

- $C(q, \dot{q}) \in \mathbb{R}^{n}$ is the vector expressing the Coriolis and the centrifugal forces,

- $G(q) \in \mathbb{R}^{n}$ is the gravitational forces vector,

- $u=\tau \in \mathbb{R}^{n}$ denotes the external control torques and forces vector, applied at each joint.

\section{Sliding mode Control concept}

Consider a nonlinear affine system presented as:

$\dot{x}(t)=\varphi(x)+\gamma(x) u(t)$

where $x \in \mathbb{R}^{n}, \quad u \in \mathbb{R}^{m}, \quad \varphi(x) \in \mathbb{R}^{n} \times \mathbb{R}^{m}, \quad$ and $\gamma(x) \in \mathbb{R}^{n} \times \mathbb{R}^{m}$.

The $(n \times m)$ dimensional sliding manifold that is described as (3), is determined by the sliding manifold intersection $\mathrm{s}_{i}(\mathrm{x})=0$.

$$
S(x)=\left[\begin{array}{llll}
S_{1}(x) & S_{2}(x) & \ldots & S_{m}(x)
\end{array}\right]^{T}=0
$$

Here, $\mathrm{s}_{i}(\mathrm{x})$ denotes the $i^{\text {th }}$ component of the vector $\mathrm{S}(\mathrm{x})$.
First, the adequate expression of the position error is chosen as:

$$
\varepsilon=e+\lambda \int e d t
$$

where $\mathrm{e}=\mathrm{q}-\mathrm{q}_{d}$ and $\lambda$ is a positive scalar gain.

Taking into consideration the fact that the static error can not be completely canceled, especially in the case of uncertainties and parametric variations, the selected integral term added to the error expression occurs to push this static error to be totally canceled. Thus, consider the integral sliding surface that can be written as:

$s=\dot{\varepsilon}+\lambda \varepsilon$

In the present sliding mode study, we are especially interested in the equivalent control theory. The classical approach can be summarized as follows.

\section{Equivalent control theory:}

The SMC is composed first by a 'nominal' part that allows the system without perturbations and parametric uncertainties, to be stabilized in a finite time. A 'discontinuous' part represents the required term to ensure the maintain on the sliding surface. In order to satisfy the control objective, the controller can be expressed as:

$u=u_{e q}+\Delta u$

The equivalent control can be designed as follows. When the system reaches the sliding surface, we have $\mathrm{s}(\mathrm{x})=0$, and then $\dot{s}(x)=0$. As results from the previous expressions we can obtain:

- Based on the sliding mode expression,

$\dot{s}(x)=\frac{\partial s}{\partial x}[\varphi(x)+\gamma(x) u(t)]=0$

Assuming that matrix $\frac{\partial s}{\partial x} \gamma(x)$ is regular, which

represents the intrinsic condition for the existence of sliding mode control, the associated equivalent control is written as:

$u_{e q}=-\left(\frac{\partial s}{\partial x} \gamma(x)\right)^{-1} \frac{\partial s}{\partial x} \varphi(x)$

The resulting dynamics of the system on the sliding surface $S(x)=0$ is described by:

$\dot{x}=\left[I-\gamma\left(x_{e q}\right)\left(\frac{\partial s}{\partial x} \gamma(x)\right)^{-1} \frac{\partial s}{\partial x}\right] \varphi(x)$

Besides, by adopting the simple sliding mode approach, the discontinuous term can be chosen as:

$\Delta u=-\left(\frac{\partial s}{\partial x} \gamma(x)\right)^{-1} \ell \operatorname{sign}(s)$ 
where $\ell$ is a positive definite matrix. In general, the problem of such strategy is that the control law (6) presents oscillations at high frequencies, which lead to the appearance of the chattering phenomenon, because of the discontinuous nature of the control. In this case, the "signum" function can be replaced by a saturation one.

\section{Adaptative SMC design}

The major constraint to design the detailed SMC controller is the uncertainty of system parameters Such problem can be solved using an adaptive approach, as presented in Fig. 1. Thus, consider that the physical parameters of the manipulator robot are ill known or ill defined, and assume that the full state of the robot is available.
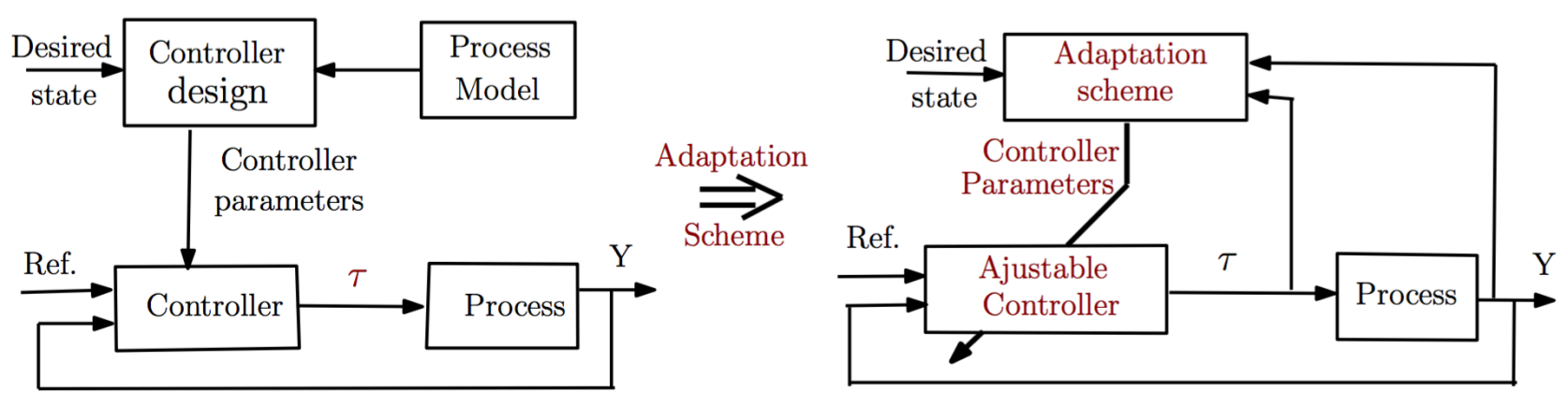

Fig. 1. (a) Principle of the controller design (b) Principle of the adaptive control version

To this end, consider the expression of the parameter estimation error $\tilde{\theta}_{i}$ :

$$
\tilde{\theta}_{i}=\hat{\theta}_{i}-\theta_{i}
$$

where:

- $\theta_{i} \in \mathbb{R}$ is an unknown constant parameters vector,

- $\hat{\theta}_{i}$ represents the estimated value of the unknown parameters vector.

Besides, assume that the unknown vector $\theta_{i}$ is constant or it has slow variations, and taking into account expression (11), we deduce that $\dot{\theta}_{i} \simeq 0$. Consequently:

$\dot{\tilde{\theta}}_{i}=\dot{\hat{\theta}}_{i}$

Therefore, the certainty equivalence form of $\theta_{i}$ is employed in such a way that it is replaced by the parameter estimate vector $\hat{\theta}_{i}$.

The differential of function $s$ with respect to time can be expressed as:

$\dot{s}=f(\theta)+g(\theta) u$

where functions $f(\theta)$ and $g(\theta)$ depend also on the state vector $x$. The expression of the equivalent control is:

$$
u_{e q}(\theta)=-[g(\theta)]^{-1} f(\theta)
$$

assuming that matrix $g(\theta)$ is regular as it has been defined above. However, vector $\theta$ is unknown, then the control expression (6) is modified in this case to be expressed as:

$$
u=u_{e q}(\hat{\theta})+\Delta u(\hat{\theta})
$$

where the discontinuous term of the control is chosen as:
$\Delta u(\hat{\theta})=-[g(\hat{\theta})]^{-1} \ell \operatorname{sign}(s)$

where $\ell=\operatorname{diag}\left[\ell_{1} \ldots \ell_{m}\right]$ is a positive diagonal matrix.

By substituting (14) into (13), we obtain the following expressions:

$$
\begin{aligned}
& \dot{s}=f(\theta)+g(\theta)\left[u_{e q}(\hat{\theta})+\Delta u(\hat{\theta})\right] \\
& =g(\tilde{\theta})\left[u_{e q}(\tilde{\theta})-u_{e q}(\theta)\right]+g(\tilde{\theta}) \Delta u(\hat{\theta}) \\
& +[g(\theta)-g(\hat{\theta})]\left[u_{e q}(\hat{\theta})-u_{e q}(\theta)\right]+[g(\theta)-g(\hat{\theta})] \Delta u(\hat{\theta})
\end{aligned}
$$

Then, and considering Taylor expansions we obtain:

$$
\begin{aligned}
& u_{e q}(\hat{\theta})-u_{e q}(\theta)=\sum_{i} \frac{\partial u_{e q}}{\partial \theta_{i}} \tilde{\theta}_{i}+o(\tilde{\theta})^{2} \\
& g(\theta)-g(\hat{\theta})=-\sum_{i} \frac{\partial g}{\partial \theta_{i}} \tilde{\theta}_{i}+o(\tilde{\theta})^{2}
\end{aligned}
$$

$[g(\theta)-g(\hat{\theta})]\left[u_{e q}(\hat{\theta})-u_{e q}(\theta)\right]=o(\tilde{\theta})^{2}$

The substitution of (17), (18) and (19) into ( $=g(\tilde{\theta})\left[u_{e q}(\tilde{\theta})-u_{e q}(\theta)\right]+g(\tilde{\theta}) \Delta u(\hat{\theta})$

$+[g(\theta)-g(\hat{\theta})]\left[u_{e q}(\hat{\theta})-u_{e q}(\theta)\right]+[g(\theta)-g(\hat{\theta})] \Delta u(\hat{\theta})$

yields:

$\dot{s}=\sum_{i}\left[g(\hat{\theta}) \frac{\partial u_{e q}}{\partial \theta_{i}}-\frac{\partial g}{\partial \theta_{i}} \Delta u(\hat{\theta})\right] \hat{\theta}_{i}+g(\hat{\theta}) \Delta u(\hat{\theta})+o(\tilde{\theta})^{2}$

Theorem 1:

The control law (14) with the following adaptive law: 
$\dot{\hat{\theta}}_{i}=-\rho_{i} s^{T}\left[g(\hat{\theta}) \frac{\partial u_{e q}}{\partial \theta_{i}}-\frac{\partial g}{\partial \theta_{i}} \Delta u(\hat{\theta})\right]$

stabilize the closed loop system (1), where $\rho_{i}$ is a positivedefinite control gain, for $\mathrm{i}=1,2$..

\section{Proof:}

Let's consider the following Lyapunov function candidate as:

$$
V=\frac{1}{2}\left[s^{T} s+\sum_{i} \frac{1}{\rho_{i}} \hat{\theta}_{i}^{2}\right]
$$

By differentiating it with respect to time, we obtain:

$$
\begin{aligned}
& \dot{V}=[\underbrace{\left.s^{T}(\hat{\theta}) \frac{\partial u_{e q}}{\partial \theta_{i}}-\frac{\partial g}{\partial \theta_{i}} \Delta u(\hat{\theta})\right)+\frac{1}{\rho_{i}} \dot{\hat{\theta}}_{i}}_{=0}] \hat{\theta}_{i}+ \\
& s^{T} g(\hat{\theta}) \Delta u(\hat{\theta})+o(\tilde{\theta})^{2}
\end{aligned}
$$

Thus, considering the adaptive condition (21), and replacing (15) into (23) we obtain:

$$
\begin{aligned}
& \dot{V}=-s^{T} l \operatorname{sign}(s)+o(\tilde{\theta})^{2} \\
& =-\sum_{i} l_{i}\left|s_{i}\right|+o(\tilde{\theta})^{2} \leq 0
\end{aligned}
$$

This proves the stability of the closed-loop system.

\section{Application to a 3 DOF SCARA robot}

In order to prove the efficiency of the proposed approach, consider a three degrees of freedom "SCARA" manipulator system controlled by the simple and the adaptive SMC approach applied for a motion control task.

The purpose of the controller is to ensure an accurate trajectory tracking and to force both position and velocity errors to be canceled. Table 1 mentions the initial position of the SCARA robot articulations initially at rest, and their dynamic parameters.

Table 1. Joints initiation and parameters

\begin{tabular}{c|c|c|c}
\hline Articulation & Initial position & $\begin{array}{c}\text { Mass } \\
(\mathbf{k g})\end{array}$ & $\begin{array}{c}\text { Length } \\
(\mathbf{m})\end{array}$ \\
\hline$q_{1}$ & $\pi / 5$ & 8 & 0.4 \\
$q_{2}$ & $\pi / 5$ & 6 & 0.3 \\
$q_{3}$ & 0.4 & 0.5 & 0.3 \\
\hline
\end{tabular}

Consider that the desired trajectory to be tracked has periodic form like:

$$
q_{d}(t)=\left[\begin{array}{c}
q_{d 1}(t) \\
q_{d 2}(t) \\
q_{d 3}(t)
\end{array}\right]=\left[\begin{array}{c}
\frac{\pi}{6} \sin 1.4 \pi t \\
\frac{\pi}{4} \sin 2 \pi t \\
0.2 \operatorname{sign}(2.5-t)
\end{array}\right]
$$

Here, an important remark should be noted concerning the gain values selection. In effect, an adequate gain selection should guarantee a sufficient rapidity and stability of the system, while avoiding the exceeding of actuators limitations, i.e. $\left(|\tau| \leq \tau_{\max }\right)$.

The evolution of the velocity and trajectory tracking behaviors with respect to time of the adaptive SMC are shown in Fig. 6, whereas the generalized error vector $s(x)$ and the tracking error are presented in Fig. Error! Reference source not found. and the estimated mass value evolution is identified in Fig.Fig 8.
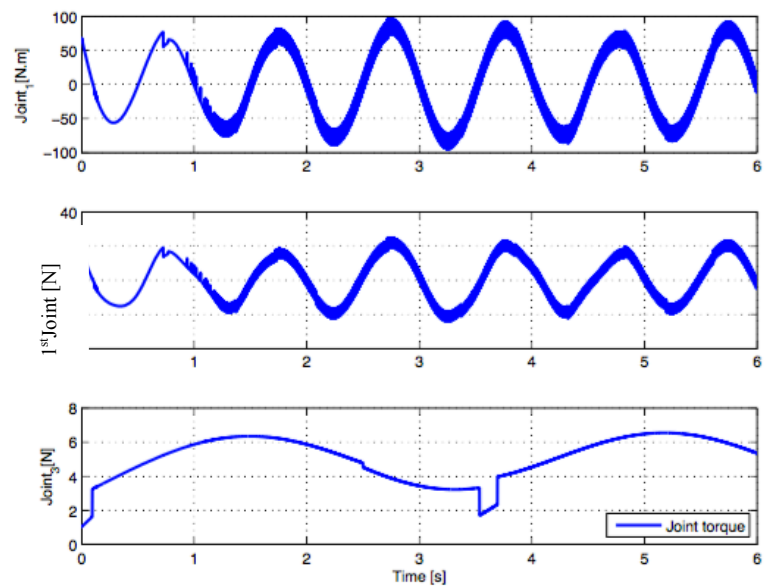

a)
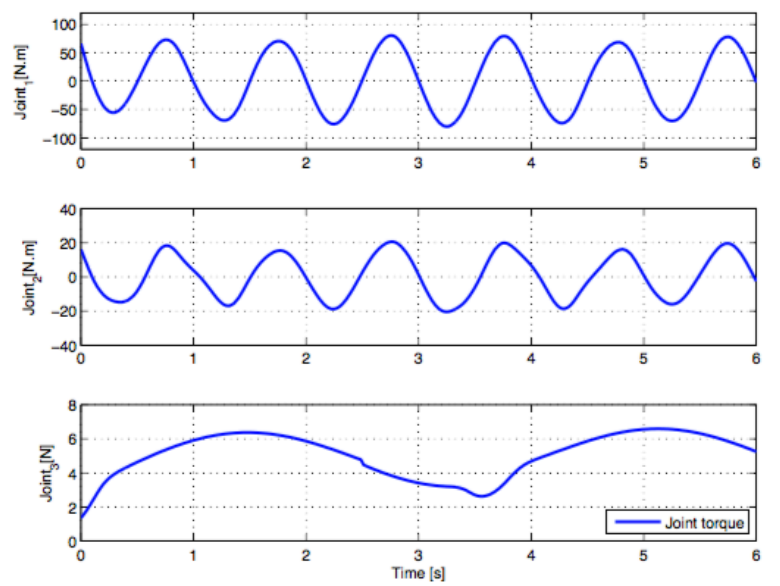

b)

Fig. 2 The SMC torques in the case of: (a) The "signum" function, (b) The "saturation" function. 

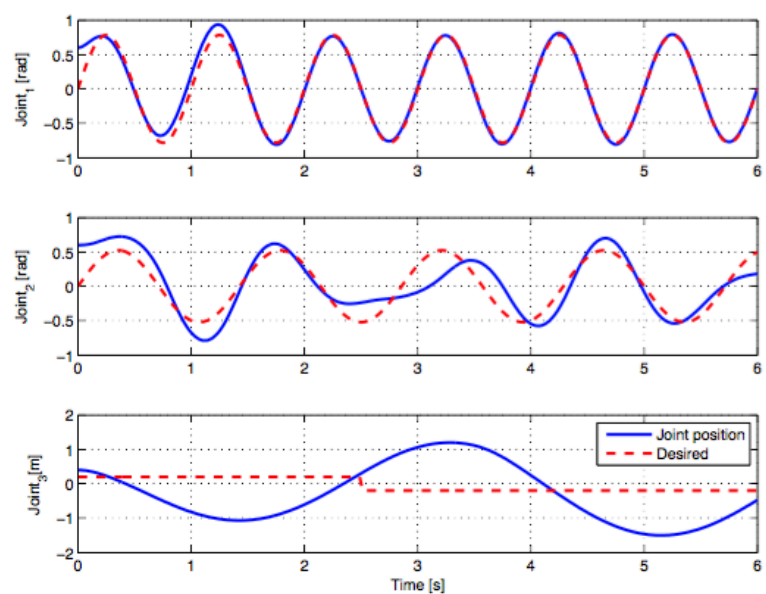

a)
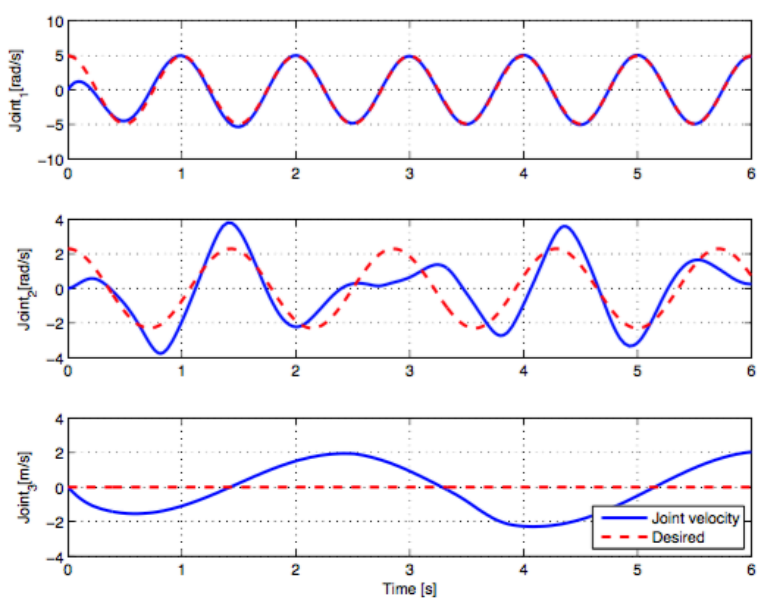

b)

Fig. 3. (a) Trajectory tracking of the SMC, (b) Velocities evolution of the SMC.
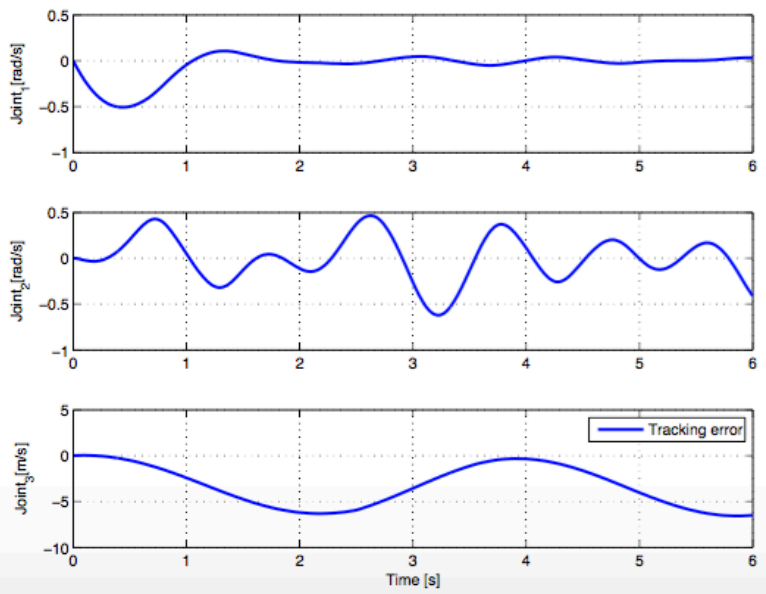

a)
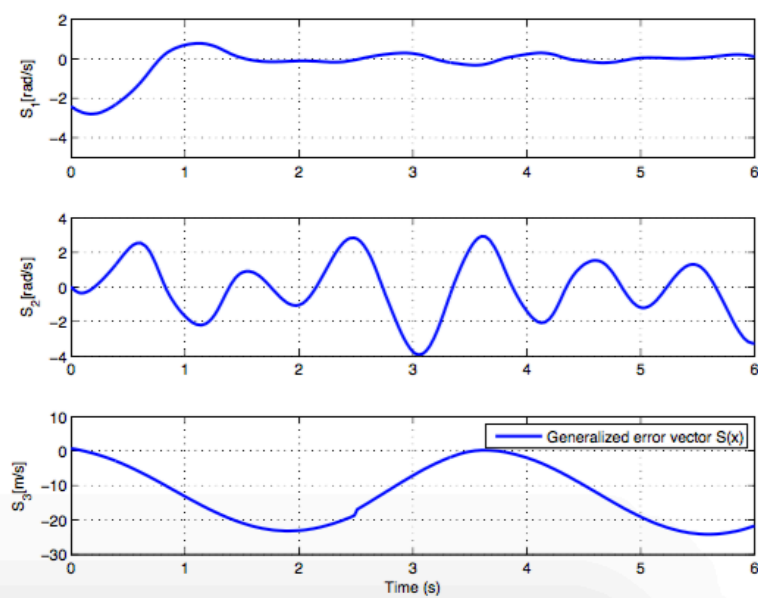

b)

Fig. 4. (a) The SMC tracking error evolutionn, (b) The generalized error vector of the SMC.
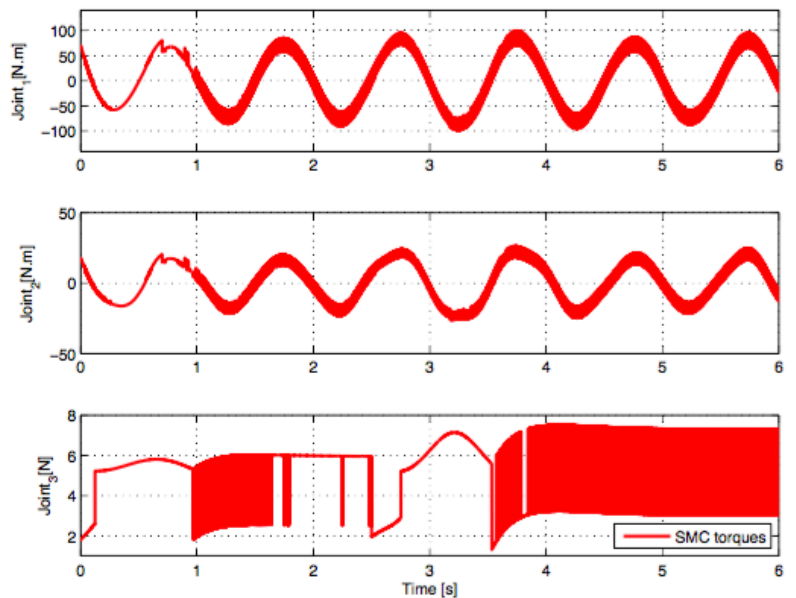

a)
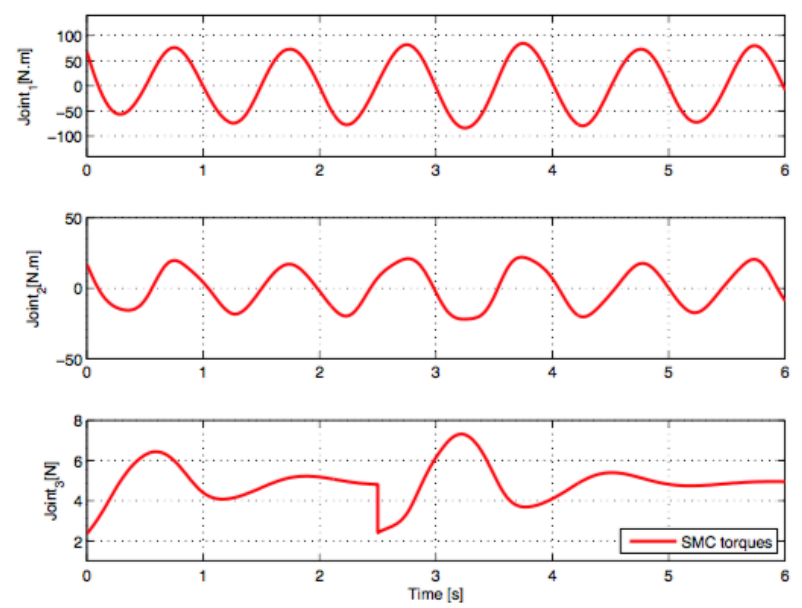

b)

Fig. 5: Adaptive SMC torques in the case of: (a) "signum" function, (b) "saturation" function. 

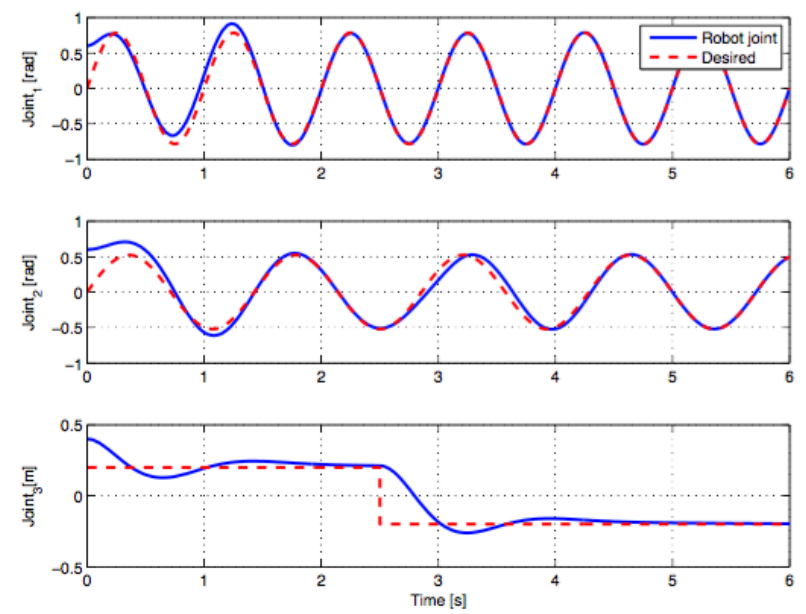

a)
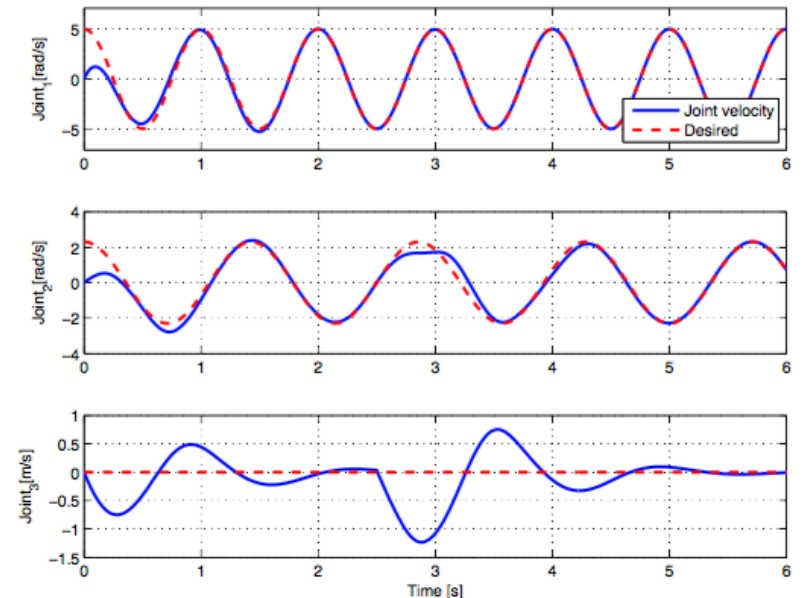

b)

Fig 6: (a) Trajectory tracking of the adaptive SMC, (b) Velocities evolution of the adaptive SMC.
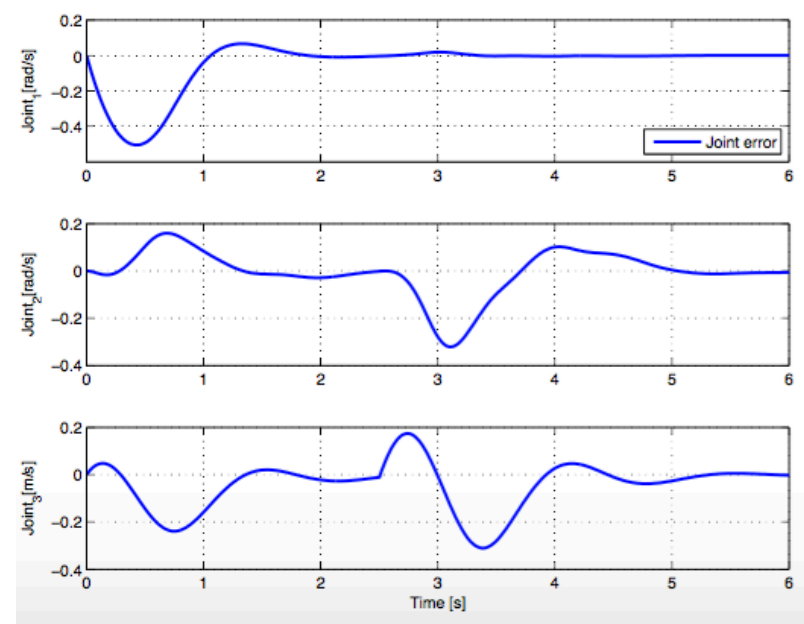

a)
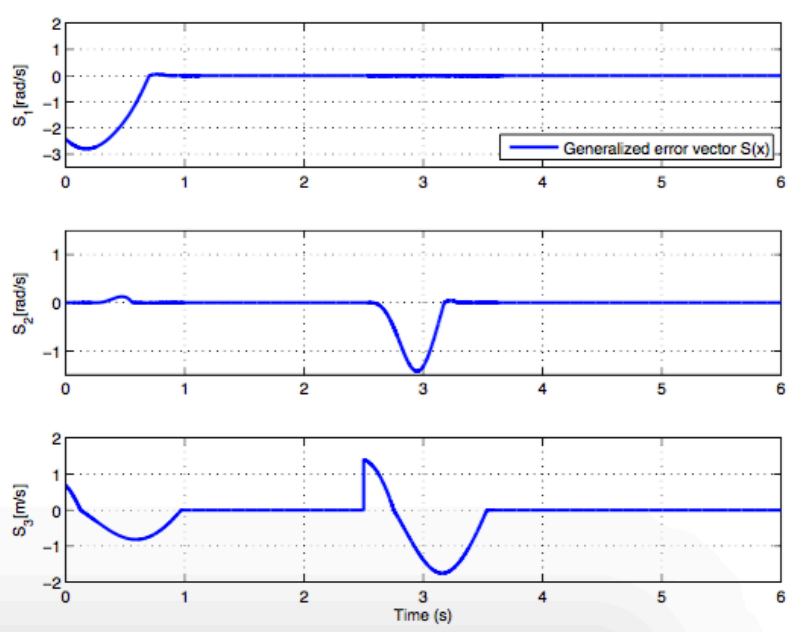

b)

Fig 7: (a) Tracking errors of the adaptive SMC, (b) Generalized error vectors of the adaptive SMC

\section{Discussion}

The proposed adaptive SMC simulation results are compared to the simple SMC, firstly developed under uncertain situations tainted by parametric variations, where the mass of the third link is assumed to be unknown.

Figure 5 presents the torques behavior of the SMC approach, where the "signum" function has been replaced by a "saturation" one. Considering the trajectory tracking and velocity behaviors represented in Fig. 6a and in Fig. 6b, controller results reveal a failing trajectory tracking task, predominately affecting the third articulation attained by the uncertain mass variation, and disrupting to a lesser extent the second joint. Consequently, tracking errors shown in Fig. 7a present high error values. Considering the generalized error vector behavior, only the first joint has obtained a sliding surface within $1.5 \mathrm{~s}$, but other joints controls have been not able to nullify associated generalized error vectors and to create their correspondant sliding surfaces.

Consequently, and as a solution to resolve the parametric variations problem faced by the simple SMC, an adaptive control law has been incorporated in the system control to enhance previous results. Simulation results of the adaptive SMC yields a good trajectory tracking evolution of all robot joints, despite the presence of parameter uncertainties inflicting the system. Thus, the uncertain mass value of the third joint has been successfully settled as represented in Fig. Fig 8, where the accurate mass value has been attained and then stabilized in its right value, that is $m_{3}=0.5 \mathrm{~kg}$. Furthermore, generalized errors evolution shown in Fig. 5 rapidly attains the zero value to establish therefore the convenient sliding surface.

Through the performed comparison between two controllers, it has been proved that the proposed adaptive SMC ensures good tracking behavior with low errors values shown in Fig. 7a, in the presence of parameter uncertainties. 


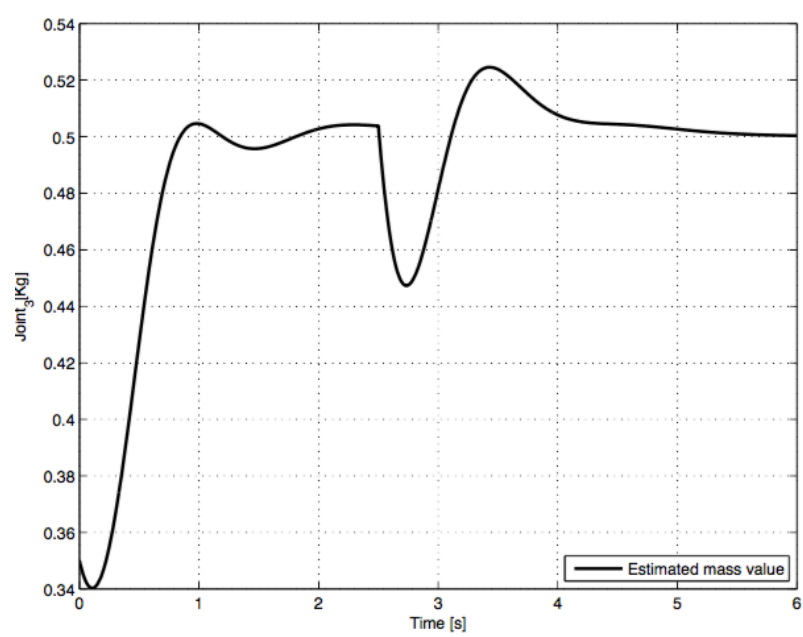

Fig 8: Estimated mass value of the adaptive SMC

\section{Conclusion}

The efficiency of the proposed strategy has been confirmed by controlling a SCARA robot manipulator in a trajectory tracking task. The first algorithm is based on the evaluation of the uncertainties impact, by implementing simple SMC concept for the robot motion control. It has been clear that the SMC could not achieve required performances in the trajectory tracking task. Indeed, while the third joint mass value is supposed to be unknown, the parametric variation disrupts almost the whole system operating. So, aiming to deal with physical and environmental perturbations, and by the incorporation of the adaptive approach, simulations yield satisfactory control stability and good trajectory tracking performances while considering the same parameter fluctuation conditions. Thus, simulation results illustrate a large difference in the controller acting between the simple and the adaptive SMC approach, that proves the superiority of the adaptive version in guaranteeing robustness against parameter variations and high tracking precision in presence of unexpected disruptive conditions. Along with the robust parametric variation resistance, the prior knowledge condition about the uncertainty limits, that is required for the traditional SMC concept becomes no longer necessary.

So, the present study affirms the ability of the proposed controller for application to highly complex nonlinear uncertain systems like a robotic manipulator.

\section{References}

1. Emna Kolsi Gdoura, Moez Feki and Nabil Derbel. "Sliding mode control of a hydraulic servo system position using adaptive sliding surface and adaptive gain." International Journal of Modelling, Identification and Control, Vol. 23, no. 3, pp. 248-259, 2015.

2. Kapoor, Neha, and Jyoti Ohri. "Fuzzy Sliding Mode Controller (FSMC) with Global Stabilization and Saturation Function for Tracking Control of a Robotic Manipulator." Journal of Control and Systems Engineering Vol. 1, no. 2, pp. 50, 2013.

3. Goléa, Noureddine, Ghania Debbache, and Amar Goléa. "Neural network-based adaptive sliding mode control for uncertain nonlinear MIMO systems." International Journal of Modelling, Identification and Control Vol. 16, no. 4, pp. 334-344, 2012.

4. Kapoor, Neha, and Jyoti Ohri. "Integrating A Few Actions For Chattering Reduction And Error Convergence In Sliding Mode Controller In Robotic Manipulator." International Journal of Engineering Research and Technology (IJERT), Vol. 2 Issue 5, May - 2013.

5. A. Levant, "Chattering Analysis", IEEE Trans. on Automatic Control, Vol. 55, June, 2010.

6. A.A. Al Rawi, S. A. Alobaidi, R. B. Ahmad and Y. Abid, "Evaluating Effects of Chattering on the Sliding Mode System", IJCSI International Journal of Computer Science, vol. 10, March, 2013.

7. Huang, Ying-Jeh, Tzu-Chun Kuo, and Shin-Hung Chang. "Adaptive sliding-mode control for nonlinearsystems with uncertain parameters." Systems, Man, and Cybernetics, Part B: Cybernetics, IEEE Transactions on Vol. 38, no. 2, pp. 534-539, 2008.

8. Xueli Wu, Yang Li, Jianhua Zhang and Quanmin Zhu. "Sliding mode control for neutral systems with uncertain parameters." International Journal of Modelling, Identification and Control, Vol. 21, Issue 1, pp. 65-71, 2014.

9. A. G/E Abera, B. Bandyopadhyay and S. Janardhanan, "Digital Redesign of Sliding Mode Control Algorithms Using Saturation Function: State and Multirate Output Feedback Approach", Proceedings of the $45^{\text {th }}$ IEEE Conference on Decision and Control, Manchester, USA, December 13-15, 2006.

10. F. Abdelhedi, Y. Bouteraa and H. Medhaffar, "Distributed sliding mode control of cooperative robotic manipulators", $10^{\text {th }}$ international multi-conference on Systems, Signals and Devices (SSD), 2013.

11. S. Islam, X. P. Liu, and A. El Saddik, "Adaptive sliding mode control of unmanned four rotor flying vehicle",International Journal of Robotics and Automation, 2013.automatic control, Vol. 57, April, 2012.

12. L. Fridman, Sliding Mode Enforcement after 1990: Main Results and Some Open Problems. "Sliding Modes after the First Decade of the $21^{\text {st }}$ Century", 412,Springer Verlag: Berlin, pp. 3-57, 2012. 